\title{
Compounds with Distinct Targets Present Diverse Antimicrobial and Antibiofilm Efficacy against Candida albicans and Streptococcus mutans, and Combinations of Compounds Potentiate Their Effect
}

\author{
Carmélia Isabel Vitorino Lobo (D), Ana Carolina Urbano de Araújo Lopes (D) and Marlise Inêz Klein * (D) \\ Department of Dental Materials and Prosthodontics, School of Dentistry, São Paulo State University (Unesp), \\ Araraquara. Rua Humaitá, 1680, Araraquara, São Paulo 14801-903, Brazil; carmelialobo@gmail.com (C.I.V.L.); \\ carolina.urbano@unesp.br (A.C.U.d.A.L.) \\ * Correspondence: marlise.klein@unesp.br; Tel.: +55-16-3301-6410
}

Citation: Lobo, C.I.V.; Lopes, A.C.U.d.A.; Klein, M.I. Compounds with Distinct Targets Present Diverse Antimicrobial and Antibiofilm

Efficacy against Candida albicans and Streptococcus mutans, and

Combinations of Compounds Potentiate Their Effect. J. Fungi 2021, 7,340. https://doi.org/10.3390/ jof7050340

Academic Editors: Célia F. Rodrigues and Jesus A. Romo

Received: 31 March 2021

Accepted: 25 April 2021

Published: 28 April 2021

Publisher's Note: MDPI stays neutral with regard to jurisdictional claims in published maps and institutional affiliations.

Copyright: (c) 2021 by the authors. Licensee MDPI, Basel, Switzerland. This article is an open access article distributed under the terms and conditions of the Creative Commons Attribution (CC BY) license (https:/ / creativecommons.org/licenses/by/ $4.0 /)$.
Abstract: Candida albicans and Streptococcus mutans interact synergistically in biofilms associated with a severe form of dental caries. Their synergism is driven by dietary sucrose. Thus, it is necessary to devise strategies to hinder the development of those biofilms and prevent cavities. Six compounds [ $t t$-farnesol (sesquiterpene alcohol that decreases the bacterium acidogenicity and aciduricity and a quorum sensing fungal molecule), myricetin (flavonoid that interferes with $S$. mutans exopolysaccharides production), two 2'-hydroxychalcones and 4'-hydroxychalcone (intermediate metabolites for flavonoids), compound 1771 (inhibitor of lipoteichoic synthase in Gram-positive bacteria)] with targets in both fungus and bacterium and their products were investigated for their antimicrobial and antibiofilm activities against single-species cultures. The compounds and concentrations effective on single-species biofilms were tested alone and combined with or without fluoride to control initial and pre-formed dual-species biofilms. All the selected treatments eliminated both species on initial biofilms. In contrast, some combinations eliminated the bacterium and others the fungus in pre-formed biofilms. The combinations 4 '-hydroxychalcone+tt-farnesol+myricetin, 4 '-hydroxychalcone+tt-farnesol+fluoride, and all compounds together with fluoride were effective against both species in pre-formed biofilms. Therefore, combinations of compounds with distinct targets can prevent $C$. albicans and S. mutans dual-species biofilm build-up in vitro.

Keywords: biofilm; Candida albicans; Streptococcus mutans; extracellular matrix; antimicrobial agents; antibiofilm agents

\section{Introduction}

Several human diseases are caused by dysbiotic biofilms, including tooth decay, periodontal diseases, and oral candidiasis [1]. Candida albicans is an opportunistic species that, when associated with Streptococcus mutans, contributes to forming a complex and organized biofilm that is more tolerant to environmental stresses, including antimicrobial [2]. The interaction between these two species is synergistic in the presence of dietary sucrose and leads to severe dental caries lesions [3]. Therefore, it is necessary to devise strategies to hinder the development of those biofilms.

Within the complex oral microbiota, $S$. mutans is a producer of the extracellular matrix and modulates cariogenic biofilm formation when sucrose from the host's diet is available [4]. This bacterium is acidogenic and aciduric but not the most numerous species in the mouth, and there are other acidogenic and aciduric microorganisms [5,6]. However, its exoenzymes glucosyltransferases (Gtfs) and fructosyltransferase (Ftf) use sucrose as a substrate for the synthesis of exopolysaccharides ( $\alpha$-glucans and fructans), important components of biofilm construction [4]. Gtfs also adsorb on the surface of other oral microorganisms, converting them into glucan producers [4]. C. albicans is one of the microorganisms to which Gtfs 
binds and form high amounts of exopolysaccharides [7]. This fungus is also acidogenic and aciduric [8], and oral biofilms could serve as reservoirs for it.

The extracellular matrix of $C$. albicans biofilms contains extracellular DNA, $\beta$-glucans, mannans, proteins, and lipids [9-12]. This matrix has been associated with resistance against antifungals $[13,14]$. Moreover, the biogenesis of this matrix is coordinated extracellularly, reflecting cooperative actions in the biofilm community [14]. Therefore, the production of exopolysaccharides synthesized on surfaces in situ allows adhesion and microbial accumulation [4] and contributes to the construction of the 3D matrix that surrounds and supports cells, forming an environment with acidic niches and limited diffusion [6,15]. Thus, strategies that control the matrix formation could prevent pathogenic biofilms development [13] and perhaps control the amount of C. albicans in oral biofilms that could flourish when conditions are favorable.

The therapeutic modalities for controlling dental biofilm formation are still limited. Chlorhexidine is a broad-spectrum antimicrobial agent that suppresses microorganisms levels in saliva but is not effective against mature biofilms, and its daily and continuous use is not recommended [16]. Fluoride is the mainstay of caries prevention, but its protection against the disease is incomplete [17]. Therefore, an attractive and superior strategy would be to use or include bioactive agents targeting virulence factors and the mechanisms for biofilm development.

Several studies have prioritized finding new antibiofilm agents, including natural substances [18-20]. Among the promising agents, $t t$-farnesol (a membrane-targeting sesquiterpenoid) and myricetin (a flavonoid) hinder the development of cariogenic biofilm formed by S. mutans. Myricetin inhibits the gtfs gene expression and Gtfs activity, thereby hindering the exopolysaccharides synthesis in situ $[18,19] . t t$-farnesol targets the cellular membrane, affecting the tolerance of $S$. mutans to acid stress. Both agents have a moderate biocidal effect $[18,19]$, and their combination with fluoride results in fewer and less severe carious lesions [19]. In addition, $t t$-farnesol is a derivative of the sterol biosynthesis pathway in eukaryotic cells and a quorum-sensing molecule of C. albicans [21], which keeps the fungus in yeast form. However, it appears not to affect $S$. mutans in concentrations produced when both microorganisms are co-cultivated in biofilms, possible due to the thickness and amount of biomass of these biofilms [2,3]. Additionally, at concentrations above the threshold (i.e., the physiological concentration of the species), $t t$-farnesol can induce apoptosis in planktonic cultures of $C$. albicans cells [22]. Therefore, the antibiofilm effect of $t t$-farnesol and myricetin against $C$. albicans and S. mutans biofilms still needs to be investigated [23].

Hydroxychalcones are precursor metabolic intermediates for flavonoids and isoflavonoids. These agents inhibit the streptococcal Gtfs activity [24], impair S. mutans survival in planktonic culture [25]; thus, possibly impairing the structure of biofilms. In addition, flavonoids interfere with C. albicans cell wall formation, cause disruption of the plasma membrane and mitochondrial dysfunction, affect cell division, protein synthesis, and the efflux-mediated pumping system [26]. Also, synthetic hydroxychalcones were shown to have anti-Candida activity [25,27]. Nevertheless, the efficacy of hydroxychalcones on dual-species S. mutans and C. albicans biofilms is unexplored.

Also, the interference in the metabolism of lipoteichoic acids (LTA) would affect the development of biofilms by Gram-positive bacteria. The compound 1771 targets LtaS, an LTA synthase enzyme in S. aureus [28] and Enterococcus faecium [29]. This compound also hinders S. mutans biofilm formation [30]. However, the effect of compound 1771 on $C$. albicans is unknown.

Thus, considering the virulence and difficulty of controlling mature biofilms, this study evaluated the antimicrobial and antibiofilm activities of six compounds ( $t t$-farnesol, myricetin, two 2'-hydroxychalcones, $4^{\prime}$-hydroxychalcone, and compound 1771 ) against $C$. albicans and S. mutans single- and dual-species settings. 


\section{Materials and Methods}

\subsection{Experimental Design}

Antimicrobial activity was evaluated using planktonic cultures of C. albicans and S. mutans in microdilution assay to determine the minimum inhibitory concentration (MIC) and minimum fungicidal and bactericidal concentrations (MFC and MBC). Next, singlespecies biofilms were used to investigate the antibiofilm activities of compounds during initial biofilm formation $(24 \mathrm{~h}$ ) and against pre-formed biofilms (48 h). Finally, promising compounds (and their corresponding concentrations) on both species were combined to analyze the antibiofilm activity on dual-species biofilms formed by C. albicans and S. mutans on initial biofilm formation $(24 \mathrm{~h}$ ) and pre-formed biofilms $(48 \mathrm{~h})$. At that time, fluoride was also added, and groups with and without fluoride were evaluated. All tests were performed using a 96-polystyrene microplate to determine viable microbial population (colony forming units or CFU). At least three independent experiments were performed in triplicate for the antimicrobial and antibiofilm tests $(n=9)$. The data were statistically analyzed according to the factorial design of this study, considering each microplate well as a statistical block. The hypothesis was that elimination or reduction of at least $50 \%$ of microbial cells (of both species) from biofilm using the proposed agents and their combinations substantially affect the development of dual-species biofilms.

\subsection{Test Agents}

An in vitro study with $C$. albicans and $S$. mutans was conducted to investigate the antimicrobial and antibiofilm activity of six compounds: $t t$-farnesol or (E,E)-3,7,11Trimethyl-2,6,10-dodecatrien-1-ol, trans,trans-3,7,11-Trimethyl-2,6,10-dodecatrien-1-ol (Sigma-Aldrich Co., St Louis, MO, USA; Cat.\#46193; 96\% purity), myricetin or 3,5,7trihydroxy-2-(3,4,5-trihydroxyphenyl)-4H-chromen-4-one (AK Scientific, Inc., Union City, CA, USA; Cat.\#J10595; 95\% purity), three hydroxychalcones [2'-hydroxichalcone or 1-(2-hydroxyphenyl)-3-phenylprop-2-en-1-one (Angene, Hong Kong, China; Cat.\#AGNPC-015IM; 95\% purity), 2'-hydroxichalcone or (2E)-1-(2-hydroxyphenyl)-3-phenylprop2-en-1-one (AK Scientific, Inc.; Cat.\#R815; 98\% purity), 4'-hydroxichalcone or (2E)-1-(4hydroxyphenyl)-3-phenylprop-2-en-1-one (AK Scientific, Inc.; Cat.\#C135; 98\% purity)], and compound 1771 [(5-phenyl-1,3,4-oxadiazol-2-yl)carbamoyl]methyl 2-\{naphtho[2,1b]furan-1-yl\}acetate)] (UkrOrgSynthesis Ltd., Kiev, Ukraine; Cat.\#PB25353228; purity not available). The compounds were diluted with $84.15 \%$ ethanol (EtOH; Sigma-Aldrich; Cat.\#E7023) and 15\% dimethyl sulfoxide (DMSO; Sigma-Aldrich; Cat.\#D8418) to have stock solutions at $15 \mathrm{mg} / \mathrm{mL}$, except for compound 1771 that was used at $2 \mathrm{mg} / \mathrm{mL}$. The concentration for compound 1771 was lower than the other agents because of solubility issues. Also, sodium fluoride was prepared at $5000 \mathrm{ppm}$ (Sigma-Aldrich; Cat.\# 71519). These stock solutions were diluted to distinct concentrations for assays. For antimicrobial assays, the agents with stock concentration at $15 \mathrm{mg} / \mathrm{mL}$ were tested using concentrations of 1250, 1000, 500, 250, 125, 62.5, 31.25, $15.625 \mu \mathrm{g} / \mathrm{mL}$. For compound 1771 (stock concentration at $2 \mathrm{mg} / \mathrm{mL}$ ), the concentrations used were 250, 125, 62.5, $31.25,15.625,7.813,3.906,1.953,0.977,0.488,0.244 \mu \mathrm{g} / \mathrm{mL}$.

\subsection{Microbial Strain and Growth Conditions}

C. albicans SC5314 and S. mutans UA159 (serotype c; ATCC 700610) strains preserved in a freezer $-80^{\circ} \mathrm{C}$ were thaw, platted on a blood agar plate $(5 \%$ sheep's blood; Laborclin, Pinhais, PR, Brazil), and incubated $\left(48 \mathrm{~h}, 37^{\circ} \mathrm{C}, 5 \% \mathrm{CO}_{2}\right.$; Steri-Cult ${ }^{\mathrm{TM}}$ Thermo Scientific, Waltham, MA, USA). Next, five colonies of each microorganism were inoculated into a liquid culture medium (2.5\% tryptone with $1.5 \%$ yeast extract or TY, $\mathrm{pH} 7.0$; Becton, Dickinson and Company (BD), Sparks, MD, USA) containing $1 \%$ of glucose $(w / v)(\mathrm{TY}+1 \%$ glucose) and incubated $\left(16 \mathrm{~h}, 37^{\circ} \mathrm{C}, 5 \% \mathrm{CO}_{2}\right)$. After that, the starter cultures were diluted 1:20 in the same culture medium and grown until mid-log growth phase: $S$. mutans $\mathrm{OD}_{562 \mathrm{~nm}}$ $0.500( \pm 0.100)$ and C. albicans $\mathrm{OD}_{562 \mathrm{~nm}} 0.482( \pm 0.058)$ (ELISA plate reader, Biochrom Ez, Cambourne, UK). These cultures were diluted in TY $+1 \%$ glucose for each microorganism 
inoculum with $2 \times 10^{6}$ colony-forming units per milliliter $(\mathrm{CFU} / \mathrm{mL})$ for antimicrobial evaluation. However, the mid-log growth phase cultures were diluted in $\mathrm{TY}+1 \%$ sucrose $(w / v)$ to yield $2 \times 10^{6} \mathrm{CFU} / \mathrm{mL}$ for single-species antibiofilm assays.

\subsection{Antimicrobial Activity}

The antimicrobial activity was evaluated by determining the minimum inhibitory concentration (MIC) and minimum fungicidal and bactericidal concentration (MFC and MBC, respectively) using broth microdilution following the Clinical and Laboratory Standards Institute [31-33], with some modifications [34]. The compounds were evaluated according their stock concentration ranging from 0 to $1250 \mu \mathrm{g} / \mathrm{mL}$ (when stock concentration $15 \mathrm{mg} / \mathrm{mL}$ ) and 0 to $250 \mu \mathrm{g} / \mathrm{mL}$ (for compound 1771 with stock concentration at $2 \mathrm{mg} / \mathrm{mL}$ ). Of note, $0 \mu \mathrm{g} / \mathrm{mL}$ was the vehicle-control. For most of the newest compounds, MIC has been described with some caveats, as when the visual inspection and the optical density are compromised by precipitation, for example [35]. Here, most agents complexed when in contact with the culture medium, making visual inspection subjective and interfering with optical density readings, and most of them did not present a clear dose-dependent effect. Therefore, the abbreviation $\mathrm{IC}_{50}$ was defined as the inhibitory concentration of the agent that inhibited the growth of the microorganism by 50\% [34], considering microbial growth as the $\mathrm{CFU} / \mathrm{mL}$ counts. Thus, MIC abbreviation was not employed to state the outcomes.

All selected compounds [tt-farnesol, myricetin, 2'-hydroxychalcone (AGN), 2' hydroxychalcone (R815), 4'-hydroxychalcones (C135), and compound 1771 (1771)] were tested against $S$. mutans planktonic culture. However, only the effective compounds against the bacterium ( $t$-farnesol, myricetin, C135, and compound 1771) were used for C. albicans because when both species are together, they form robust biofilms mediated primarily by the exopolysaccharides produced by the bacterium in a rich-sucrose environment [3].

The inoculum culture (100 $\mu \mathrm{L}$ of bacterium or fungus) was transferred to 96 -well plates containing $\mathrm{TY}+1 \%$ glucose and distinct concentrations of agents were arranged from the highest to the lowest for a final volume of $200 \mu \mathrm{L}$ (yielding $1 \times 10^{6} \mathrm{CFU} / \mathrm{mL}$ for each species). As controls for each experiment, wells containing only culture medium, only inoculum (growth control without treatment), and inoculum plus vehicle (final concentration of $7 \% \mathrm{EtOH}$ and $1.25 \%$ DMSO) were added to rule out any effect of the vehicle on microbial cells. The assembled plates were incubated $\left(24 \mathrm{~h}, 37^{\circ} \mathrm{C}, 5 \% \mathrm{CO}_{2}\right)$, followed visual inspection (turbidity: microbial growth, clear: no growth), and $\mathrm{OD}_{562 \mathrm{~nm}}$ readings (ELISA plate reader). Next, the plates were incubated to homogenize the cultures ( $5 \mathrm{~min}, 75 \mathrm{rpm}$, $37^{\circ} \mathrm{C}$ ) (Quimis, São Paulo, Brazil). After that, an aliquot from each well was removed for serial dilution in saline solution $(0.89 \% \mathrm{NaCl}$; Synth, Diadema, SP, Brazil), plating (undiluted and $10^{-1}$ to $10^{-5}$ ), and incubation $\left(48 \mathrm{~h}, 37^{\circ} \mathrm{C}, 5 \% \mathrm{CO}_{2}\right)$ to determine $\mathrm{CFU} / \mathrm{mL}$ quantification and inhibitory concentration $\left(\mathrm{IC}_{50}\right)$. The $\mathrm{MBC}$ and $\mathrm{MFC}$ were measured by $\mathrm{CFU} / \mathrm{mL}$ count and defined as the lowest compound concentration that inhibited microbial growth (or absence of colony growth on agar). However, for some compounds that may target the extracellular matrix production in biofilms, the concentrations that inhibited at least $50 \%$ of microbial growth (i.e., $50 \%$ of $\mathrm{CFU} / \mathrm{mL}$ reduction vs. vehicle) were considered promising antimicrobial activity $[34,35]$.

\subsection{Antibiofilm Activity}

This analysis was conducted after determining antimicrobial activity and was performed using single- and dual-species settings and different exposure to compounds: activity against initial biofilm formation $(24 \mathrm{~h})$ and pre-formed biofilms $(48 \mathrm{~h})$. For initial biofilm formation, the agents were introduced at the time $0 \mathrm{~h}$, and biofilms were evaluated at $24 \mathrm{~h}$ of development. For pre-formed biofilms, biofilms were grown for $24 \mathrm{~h}$ and then treated, being evaluated at $48 \mathrm{~h}$ of growth. Thus, it was evaluated the inhibition of biofilm formation for biofilms at $24 \mathrm{~h}$ and the eradication of biofilm growth for $48 \mathrm{~h}$-old biofilms. The measurement was considered effective when the $\mathrm{CFU} / \mathrm{mL}$ count was reduced by $50 \%$ (vs. vehicle) for 24 and 48 h-old biofilms [34,36]. 
The strains were grown and prepared using the methodology described above. However, the culture medium and inoculum of the experiments were prepared using TY $+1 \%$ sucrose. The selected compounds and their concentrations were based on antimicrobial data: C135 (from 1250 to $62.5 \mu \mathrm{g} / \mathrm{mL}$ ), myricetin (from 1250 to $125 \mu \mathrm{g} / \mathrm{mL}$ ), $t$-farnesol (from 1250 to $31.25 \mu \mathrm{g} / \mathrm{mL}$ ), and compound 1771 (from 250 to $1.953 \mu \mathrm{g} / \mathrm{mL}$ ). Previous studies evaluated the antibiofilm activity of myricetin, compound 1771 , and $t t$-farnesol for S. mutans $[18,19,30]$, but here distinct concentrations were tested. Tests with compound 1771 for C. albicans and C135 for both species will be presented for the first time.

Initially, single-species biofilm to prevent initial biofilm formation $(24 \mathrm{~h})$ were analyzed. On a 96-well plate, $100 \mu \mathrm{L}$ of final inoculum with $2 \times 10^{6} \mathrm{CFU} / \mathrm{mL}$ (for both species) were added to each well, containing test concentrations and culture medium (TY $+1 \%$ sucrose), totalizing $200 \mu \mathrm{L}\left(1 \times 10^{6} \mathrm{CFU} / \mathrm{mL}\right)$. Controls of experiments were also added (wells containing only culture medium, wells containing only the inoculum of the experiment, and wells containing the inoculum plus vehicle or $0 \mu \mathrm{g} / \mathrm{mL}$ ). The plate was incubated $\left(24 \mathrm{~h}, 37^{\circ} \mathrm{C}, 5 \% \mathrm{CO}_{2}\right)$. After incubation, visual inspection was performed, followed by orbital incubation $\left(5 \mathrm{~min}, 75 \mathrm{rpm}, 37^{\circ} \mathrm{C}\right)$. The remaining biofilms on the wells were rinsed three times with $200 \mu \mathrm{L}$ of $0.89 \% \mathrm{NaCl}$ solution to remove any loose material. Next, these biofilms were scraped with pipet tips five times (5X) with $200 \mu \mathrm{L}$ of $0.89 \% \mathrm{NaCl}$, totalizing $1 \mathrm{~mL}$ of biofilm suspension (from each well). This biofilm suspension was placed in a microtube, subjected to serial dilutions $\left(10^{-1}\right.$ to $\left.10^{-5}\right)$, which were plated, as were the undiluted suspensions. The plates were incubated $\left(48 \mathrm{~h}, 37^{\circ} \mathrm{C}, 5 \% \mathrm{CO}_{2}\right)$, and the $\mathrm{CFU} / \mathrm{mL}$ was calculated. Next, the microbial growth inhibition of each concentration was compared to vehicle control.

Subsequently, prevention of build-up pre-formed biofilm (48 h) was evaluated [36]. Here, 96-well plates were assembled using $100 \mu \mathrm{L}$ final inoculum of C. albicans or $S$. mutans $\left(2 \times 10^{6} \mathrm{CFU} / \mathrm{mL}\right)$ and $100 \mu \mathrm{L}$ of $\mathrm{TY}+1 \%$ sucrose (to reach $\left.1 \times 10^{6} \mathrm{CFU} / \mathrm{mL}\right)$. The microplate was incubated $\left(24 \mathrm{~h}, 37^{\circ} \mathrm{C}, 5 \% \mathrm{CO}_{2}\right)$ without any treatment or vehicle control. After incubation and biofilm formation, visual inspection was performed, followed by culture medium removal and washing of remaining biofilms (three times with $200 \mu \mathrm{L}$ $0.89 \% \mathrm{NaCl}$ solution). Next, fresh culture medium $\mathrm{TY}+1 \%$ sucrose and test concentrations of agents or the vehicle were added. The microplate was incubated again $\left(24 \mathrm{~h}, 37^{\circ} \mathrm{C}, 5 \%\right.$ $\mathrm{CO}_{2}$ ). After incubation (when biofilms were $48 \mathrm{~h}$-old), the same processing protocol applied for $24 \mathrm{~h}$-old biofilms was conducted until obtaining $1 \mathrm{~mL}$ of biofilm suspension. The biofilm suspensions were sonicated (30 s at 7 w, Sonicator QSonica, Q125, Newtown, CT, USA), subjected to serial dilutions $\left(10^{-1}\right.$ to $\left.10^{-5}\right)$, and plated. The undiluted suspensions were also plated. The CFU/mL counts were evaluated and compared to vehicle control.

Finally, the antibiofilm activity for dual-species biofilms of C. albicans and S. mutans was also performed at 24 and $48 \mathrm{~h}$. These analyzes were performed with the same methodology used for single-species biofilms (24 and $48 \mathrm{~h}$ ). However, the inoculum of the dual-species culture was prepared with $2 \times 10^{6} \mathrm{CFU} / \mathrm{mL}$ of $S$. mutans and $2 \times 10^{4} \mathrm{CFU} / \mathrm{mL}$ of C. albicans [3] to reach $1 \times 10^{6} \mathrm{CFU} / \mathrm{mL}$ of $S$. mutans and $1 \times 10^{4} \mathrm{CFU} / \mathrm{mL}$ of $C$. albicans after adding culture medium or treatments.

The concentrations of agents with better results against each species in the singlespecies biofilm setting were selected: $125 \mu \mathrm{g} / \mathrm{mL}$ (C135 and $t$-farnesol), $500 \mu \mathrm{g} / \mathrm{mL}$ (myricetin), and $3.906 \mu \mathrm{g} / \mathrm{mL}$ (1771). Then, compounds with selected concentrations were combined with each other and with or without sodium fluoride (250 ppm) or F totalizing 16 groups. The elected combinations included groups without fluoride (C135, C135+tt-farnesol, C135+1771, C135+myricetin, C135+tt-farnesol+1771, $\mathrm{C} 135+t t$-farnesol+myricetin, C135+tt-farnesol+myricetin+1771, C135+1771+myricetin, $t t$-farnesol+myricetin, $1771+$ myricetin, $t t$-farnesol $+1771+$ myricetin) and groups with fluoride (250 ppm) (C135+fluoride, C135+tt-farnesol+fluoride, C135+1771+fluoride, $\mathrm{C} 135+$ myricetin+fluoride, $\mathrm{C} 135+t t$-farnesol+1771+myricetin+fluoride). The concentration of fluoride was selected based on the commercially available fluoride-based mouth rinse $[19,37]$. 


\subsection{Statistical Analyses}

The statistical analyses for $\mathrm{CFU} / \mathrm{mL}$ values were performed using descriptive and inferential statistics. Normality was evaluated with the Shapiro-Wilk test employing a significance level of $5 \%$. The data were non-parametric; thus, the data were evaluated with Kruskal-Wallis test, followed by Dunn's post-test, considering $\alpha=0.05$ (Prism 9 software, GraphPad Software, Inc. 2021). The microbial growth inhibition of each agent and concentration was compared to vehicle control. In addition, the $\mathrm{CFU} / \mathrm{mL}$ data were transformed to $\log _{10}$ or $\log$ to verify the $\log$ reduction.

\section{Results}

\subsection{Antimicrobial Activity}

\subsubsection{S. mutans}

Three compounds (AGN, C135, R815) complexed with the culture medium, making the visual inspection analysis inaccurate; turbidity was also present for myricetin (but in minor proportion than for the three aforementioned compounds), and compound 1771 (at concentrations equal of more than $31.25 \mu \mathrm{g} / \mathrm{mL}$ ). The observation of culture medium turbidity occurred immediately after adding the compound into the culture media, without microbial inoculation and incubation (controls per each concentration tested). The compound that did not complex with culture medium was $t$-farnesol, and the absence of visual growth was observed at $31.25 \mu \mathrm{g} / \mathrm{mL}$, which was also its $\mathrm{IC}_{50}$.

Regarding MBC, as per CLSI definition, the absence of colony growth on an agar plate was found for $t t$-farnesol and compound 1771, as $62.5 \mu \mathrm{g} / \mathrm{mL}$ and $250 \mu \mathrm{g} / \mathrm{mL}$, respectively. Thus, a potential antimicrobial activity was achieved for the compounds when the compound at a specific concentration yielded a $50 \%$ reduction of $\mathrm{CFU} / \mathrm{mL}$ counts compared to the vehicle control $\left(\mathrm{IC}_{50}\right)$, as follows.

For C135, the lowest concentration that yielded $50 \%$ reduction was $62.5 \mu \mathrm{g} / \mathrm{mL}$, but the same reduction was observed for 125,250 , and $500 \mu \mathrm{g} / \mathrm{mL}$ (Figure 1). Thus, the antibacterial activity of C135 was not dose-dependent.

There was an absence of expressive effect on growth inhibition for all concentrations of AGN and R815 (vs. vehicle). However, some concentrations of R815 showed statistical differences and some inhibition of growth at 500 and $250 \mu \mathrm{g} / \mathrm{mL}$ ( 2 and 1 log reduction, respectively), 125 and $62.5 \mu \mathrm{g} / \mathrm{mL}$ ( $0.5 \mathrm{log}$ reduction). These reductions were not dosedependent and are not within the cutoff established for an effective compound (Figure 1). Thus, AGN and R815 were not used in the downstream assays.

Three concentrations of myricetin presented statistical differences compared to the vehicle $(250,500$, and $1000 \mu \mathrm{g} / \mathrm{mL})$ but were not dose-dependent. However, a better effect was obtained for $500 \mu \mathrm{g} / \mathrm{mL}$, which was considered the $\mathrm{IC}_{50}$.

For compound 1771 , the $\mathrm{IC}_{50}$ was $7.813 \mu \mathrm{g} / \mathrm{mL}$; higher concentrations also demonstrated significative statistical differences (at least $4 \log$ reduction vs. vehicle) and, as mentioned above, $250 \mu \mathrm{g} / \mathrm{mL}$ rendered absence of $\mathrm{CFU} / \mathrm{mL}$ quantification on agar (MBC). 
C135

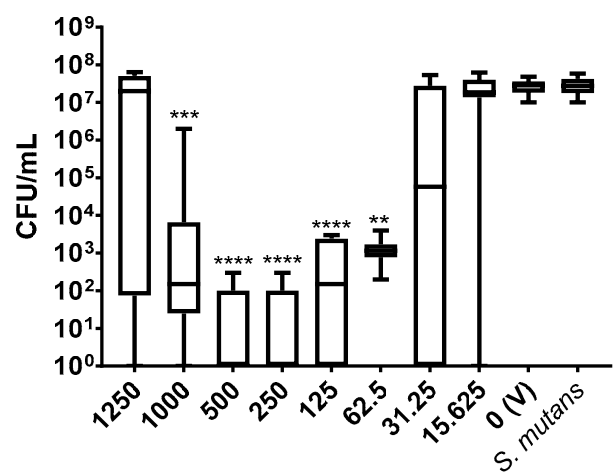

AGN

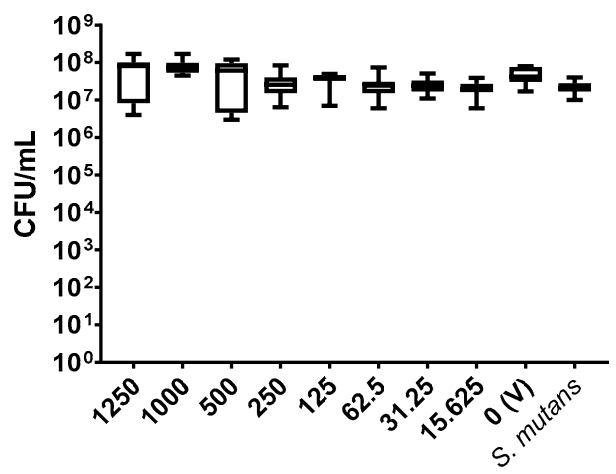

Myricetin

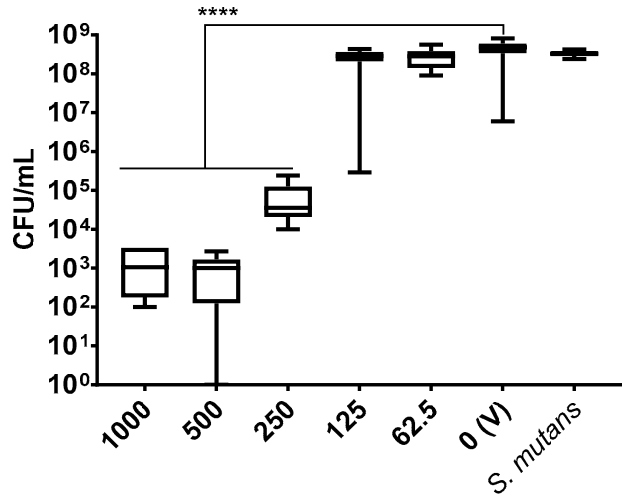

R815

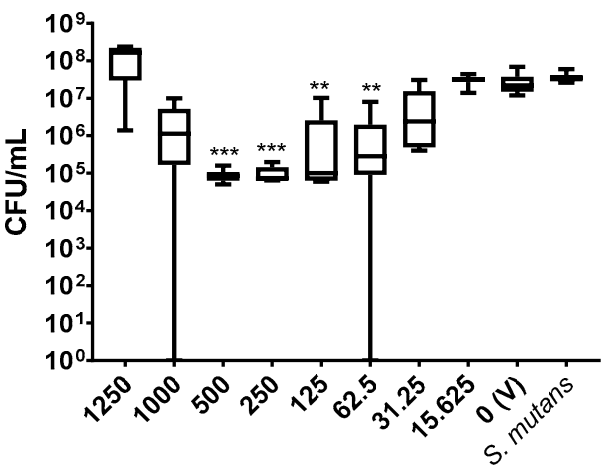

tt-farnesol

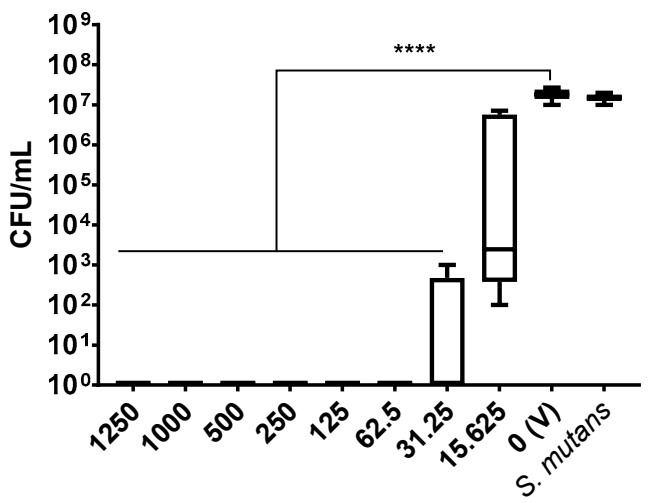

1771

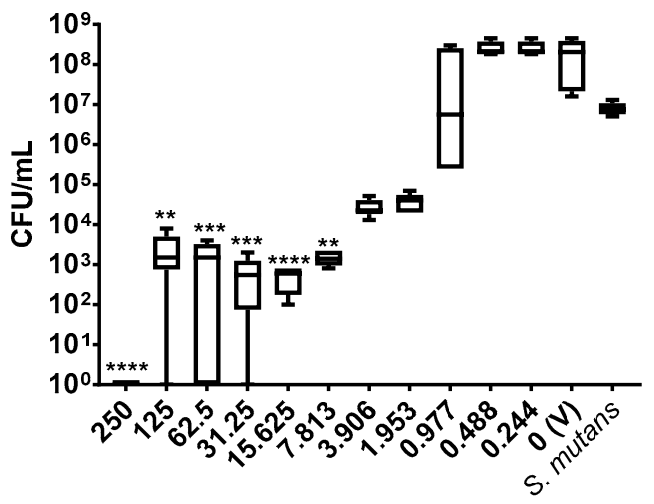

Figure 1. Antimicrobial activity of $S$. mutans using six compounds: C135, R815, AGN, $t t$-farnesol, myricetin and 1771 . Data represents median and interquartile ranges $(n=9)$. Statistical differences are represented with ${ }^{* *}(p<0.05),{ }^{* * *}(p<0.001)$, and ${ }^{* * * *}(p<0.0001)$ (Kruskal Wallis, followed by Dunn's test). The tabulated results are in Table S1.

\subsubsection{C. albicans}

The antimicrobial activity of $C$. albicans was analyzed using four agents (C135, ttfarnesol, myricetin, and compound 1771) that were selected based on the effective antimicrobial activity founded for $S$. mutans. Among them, only $t$-farnesol did not complex with the culture medium; the absence of visual growth was observed at $125 \mu \mathrm{g} / \mathrm{mL}$, which was also its $\mathrm{IC}_{50}$.

C135 and $t t$-farnesol presented a dose-dependent effect on viable fungal growth. C135 was the most effective in inhibiting fungal viability (Figure 2). All its concentrations above $31.25 \mu \mathrm{g} / \mathrm{mL}$ hindered colony growth on agar plates; thus, its $\mathrm{IC}_{50}$ and MFC were determined as $62.5 \mu \mathrm{g} / \mathrm{mL}$ (absence of colony growth on agar plates). The MFC for 
$t t$-farnesol was $1000 \mu \mathrm{g} / \mathrm{mL}$ (Figure 2). Both myricetin and compound $1771 \mathrm{did}$ not demonstrate significant antimicrobial activities as per the cutoff of $50 \%$ colony growth reduction $\left(\mathrm{IC}_{50}\right)$, although statistical differences were observed, as depicted in Figure 2.
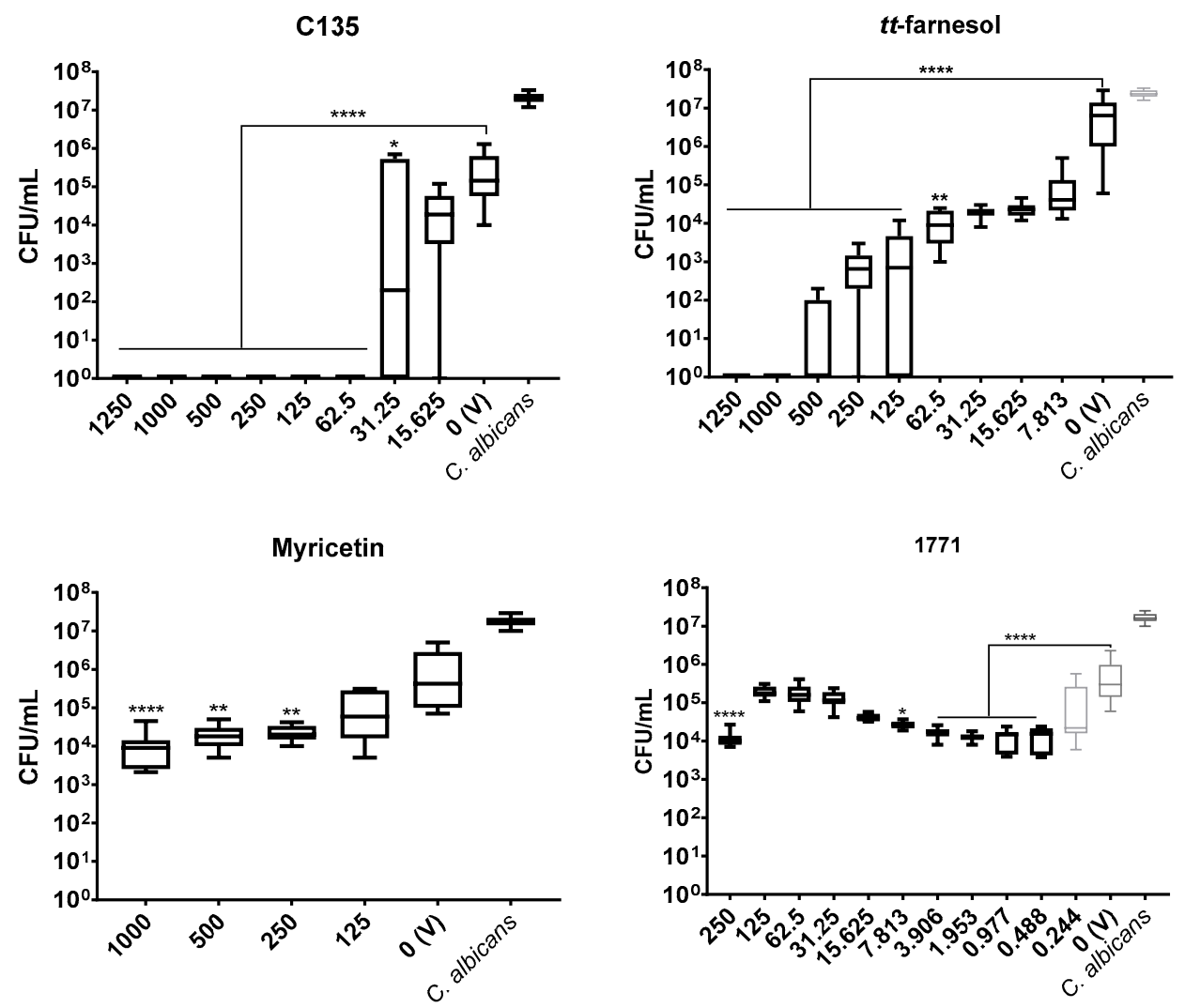

Figure 2. Antimicrobial activity of $C$. albicans with compounds: C135, $t t$-farnesol, myricetin and 1771. Data represents median and interquartile ranges $(n=9)$. Statistical differences are represented with ${ }^{*}(\mathrm{C} 135 p=0.048 ; 1771 p=0.013){ }^{* *}(p<0.05)$ and ${ }^{* * * *}(p<0.0001)$ (Kruskal Wallis, followed by Dunn's test). The tabulated results are in Table S2.

\subsection{Antibiofilm Activity}

\subsubsection{Single-Species S. mutans Biofilm}

On $24 \mathrm{~h}$ biofilm (initial biofilm formation), all concentrations of tested compounds (C135, myricetin, $t t$-farnesol, and compound 1771) demonstrated antibiofilm activity, specially myricetin and $t t$-farnesol concentrations that eliminated bacterial growth (Figure 3 ). C135 eliminated the bacterium at $62.5,125$, and $250 \mu \mathrm{g} / \mathrm{mL}$, but not at higher concentrations. Also, all concentrations of compound 1771 reduced about 5 logs of bacterium growth (vs. vehicle).

However, on $S$. mutans pre-formed biofilms $(48 \mathrm{~h})$ a greater inhibitory effect was achieved with $t t$-farnesol; where concentrations of $62.5 \mu \mathrm{g} / \mathrm{mL}$ and higher eliminated the bacterium. For C135 the best concentration was $125 \mu \mathrm{g} / \mathrm{mL}$ with 5 logs reduction (vs. vehicle). In contrast, a lower inhibitory activity was observed for myricetin and compound 1771 (although they presented statistical differences, the reduction was about 1 log vs. vehicle) (Figure 3). 

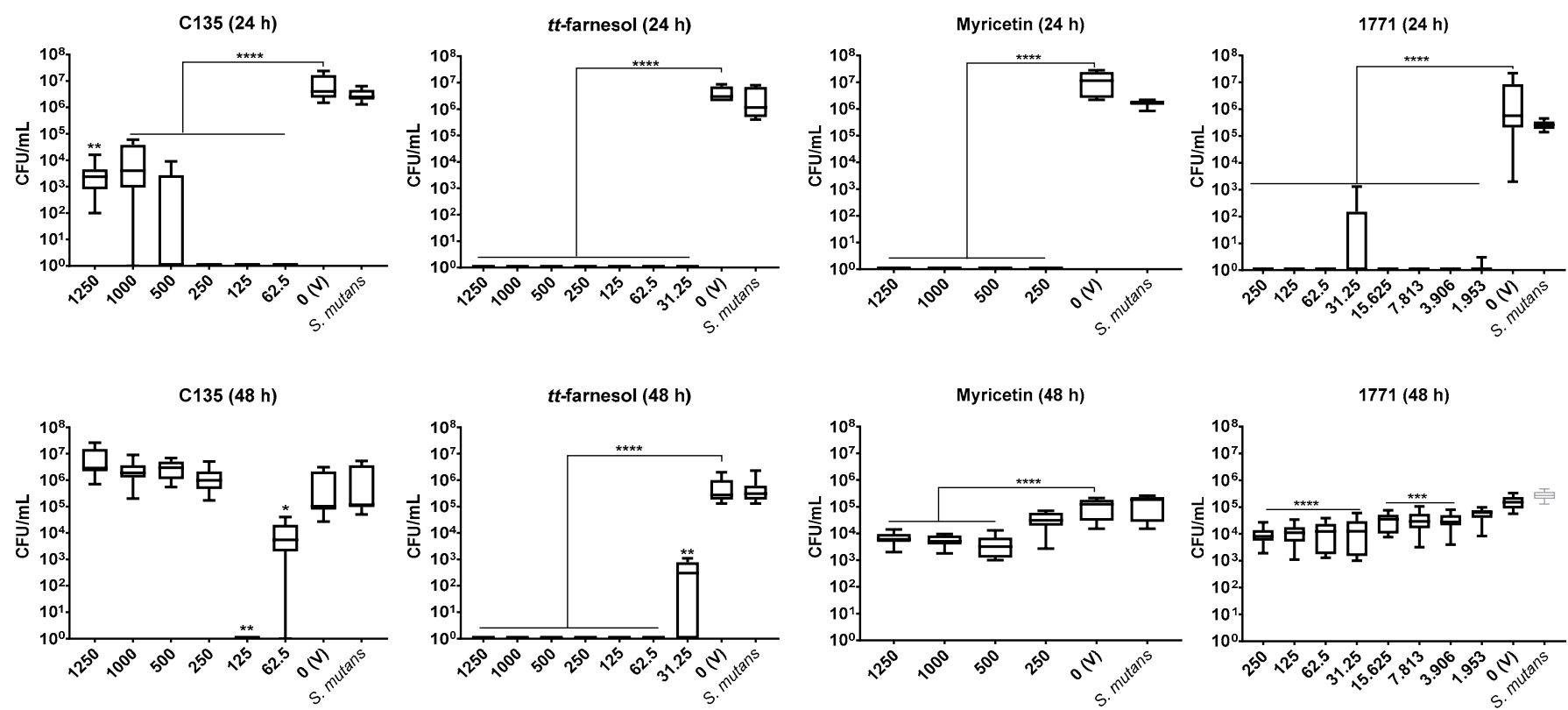

Figure 3. Antibiofilm activity of $S$. mutans with compounds: C135, $t$-farnesol, myricetin, and 1771 . On the first line are presented data of $24 \mathrm{~h}$ biofilm (initial biofilm formation); and right below are the data of pre-formed biofilms (48 h). The data represents median and interquartile ranges $(n=9)$. Statistical differences are represented with ${ }^{*}(p=0.026),{ }^{* *}(p<0.05)$, ${ }^{* * *}(p<0.001)$, and ${ }^{* * * *}(p<0.0001)$ (Kruskal Wallis, followed by Dunn's test). The tabulated results are in Table S3.

\subsubsection{Single-Species C. albicans Biofilm}

The best antibiofilm activity for C. albicans $24 \mathrm{~h}$ biofilm was observed for $\mathrm{C} 135$ and $t t$-farnesol; both eliminated the fungus, except at $31.25 \mu \mathrm{g} / \mathrm{mL}$ of $t t$-farnesol that reduced 4 logs (vs. vehicle). Compound 1771 reduced 3 logs from $3.906 \mu \mathrm{g} / \mathrm{mL}$ to higher concentrations. However, the lowest antibiofilm effect was observed for myricetin with about $1 \log$ reduction (vs. vehicle) in all tested concentrations, although statistical differences were observed (Figure 4).

The antibiofilm activity against $C$. albicans pre-formed biofilm $(48 \mathrm{~h})$ was achieved effectively only by C135. C135 eliminated the fungus at 125, 500, and $1250 \mu \mathrm{g} / \mathrm{mL}$; it also decreases CFU $/ \mathrm{mL}$ counts by $4 \operatorname{logs}(62.5 \mu \mathrm{g} / \mathrm{mL})$ and $5 \operatorname{logs}(250$ and $1000 \mu \mathrm{g} / \mathrm{mL})$ (vs. vehicle). A weak activity was observed using $t t$-farnesol, where concentrations above $125 \mu \mathrm{g} / \mathrm{mL}$ presented about $2 \log$ reduction (vs. vehicle). However, no effect was observed using myricetin and compound 1771 (except 1771 at $250 \mu \mathrm{g} / \mathrm{mL}$ with 2 logs reduction vs. vehicle) (Figure 4 ). 

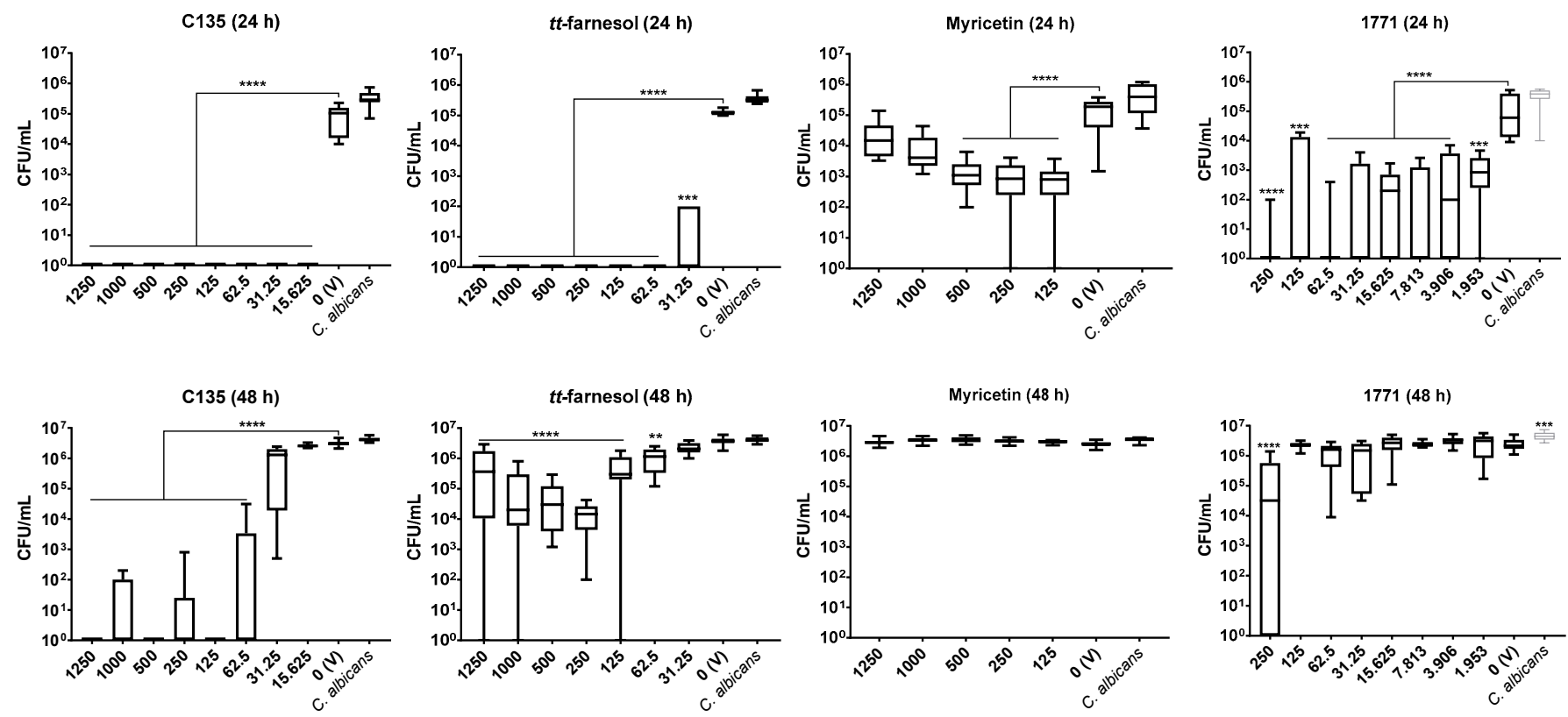

Figure 4. Antibiofilm activity of $C$. albicans with compounds: $C 135, t t$-farnesol, myricetin, and 1771 . On the first line are presented data of $24 \mathrm{~h}$ biofilm (initial biofilm formation); and right below are the data of pre-formed biofilms (48 h). The data represents median and interquartile ranges $(n=9)$. Statistical differences are represented with ${ }^{* *}(p=0.002),{ }^{* * *}(p \leq 0.0003)$, and ${ }^{* * * *}(p \leq 0.0001)$ (Kruskal Wallis, followed by Dunn's test). The tabulated results are in Table S4.

\subsubsection{Dual-Species C. albicans and S. mutans Biofilms.}

Based on the previously presented data, compounds and their most effective concentrations were selected for combinations of compounds tested on dual-species C. albicans and S. mutans biofilms (see data summarized in Table 1). The selected concentrations were: $125 \mu \mathrm{g} / \mathrm{mL}$ for $\mathrm{C} 135$ and $t t$-farnesol, $500 \mu \mathrm{g} / \mathrm{mL}$ for myricetin, and $3.906 \mu \mathrm{g} / \mathrm{mL}$ for compound 1771. In addition, C135 was used alone or combined with the other agents with and without sodium fluoride because it was the most effective agent against the fungus growth.

Table 1. Summary of antimicrobial and antibiofilm activity on single-species cultures for selection of compounds concentrations $(\mu \mathrm{g} / \mathrm{mL})$ to test against dual-species biofilms.

\begin{tabular}{|c|c|c|c|c|c|c|c|c|}
\hline \multirow{3}{*}{ Compound } & \multicolumn{4}{|c|}{ Antimicrobial Activity } & \multicolumn{4}{|c|}{ Antibiofilm Activity (Single-Species) } \\
\hline & \multicolumn{2}{|c|}{ S. mutans } & \multicolumn{2}{|c|}{ C. albicans } & \multicolumn{2}{|c|}{ S. mutans } & \multicolumn{2}{|c|}{ C. albicans } \\
\hline & $\mathrm{IC}_{50}$ & MBC & $\mathrm{IC}_{50}$ & MFC & $24 \mathrm{~h}$ & $48 \mathrm{~h}$ & $24 \mathrm{~h}$ & $48 \mathrm{~h}$ \\
\hline C135 & 62.5 & - & 62.5 & 62.5 & 62.5 & 125 & 15.625 & 125 \\
\hline$t t$-farnesol & 31.25 & 62.5 & 125 & 1000 & 31.25 & 62.5 & 62.5 & 125 * \\
\hline Myricetin & 500 & - & - & - & 250 & $500 *$ & $500 *$ & - \\
\hline 1771 & 7.813 & 250 & - & - & 3.906 & $3.906^{*}$ & $3.906^{*}$ & 250 * \\
\hline
\end{tabular}

* represent selected concentrations that did not reduce $50 \%$ of $\mathrm{CFU} / \mathrm{mL}$ but had a significative statistical reduction vs. vehicle between all tested concentrations. $\mathrm{IC}_{50}$ : the inhibitory concentration of the agent that inhibited the growth of the microorganism by $50 \%$, considering microbial growth as the $\mathrm{CFU} / \mathrm{mL}$ counts. $\mathrm{MBC}$ : minimum bactericidal concentration. MFC: minimum fungicidal concentration. 24 h: 24 h-old biofilms. 48 h: 48 h-old biofilms.

For 24 h-old biofilms (initial biofilm formation), all 16 formulations were effective, impeding the growth of both species (bacterium and fungus) (Figure 5). However, the microbial growth inhibition of pre-formed ( $48 \mathrm{~h}$-old biofilms) was different between treatments and species (Figure 5). Among the 16 formulations tested, three inhibited the growth of both species completely: C135+tt-farnesol+myricetin, C135+tt-farnesol+fluoride, and C135+tt-farnesol+1771+myricetin+fluoride (all compounds combined with fluoride). 
Furthermore, considering the total inhibition of bacterial growth in the dual-species setting, four formulations were effective (C135+tt-farnesol+1771+myricetin, C135+fluoride, C135+1771+fluoride, and C135+myricetin+fluoride). Considering the total inhibition of fungal growth in the dual-species setting, four treatments were effective (C135, C135+ttfarnesol, C135+1771, and C135+myricetin). Also, some formulations reduced at least 50\% $\mathrm{CFU} / \mathrm{mL}$ (vs. vehicle) of $S$. mutans (C135+tt-farnesol, C135+tt-farnesol+1771), or C. albicans (C135+tt-farnesol+1771+myricetin and C135+myricetin+fluoride).
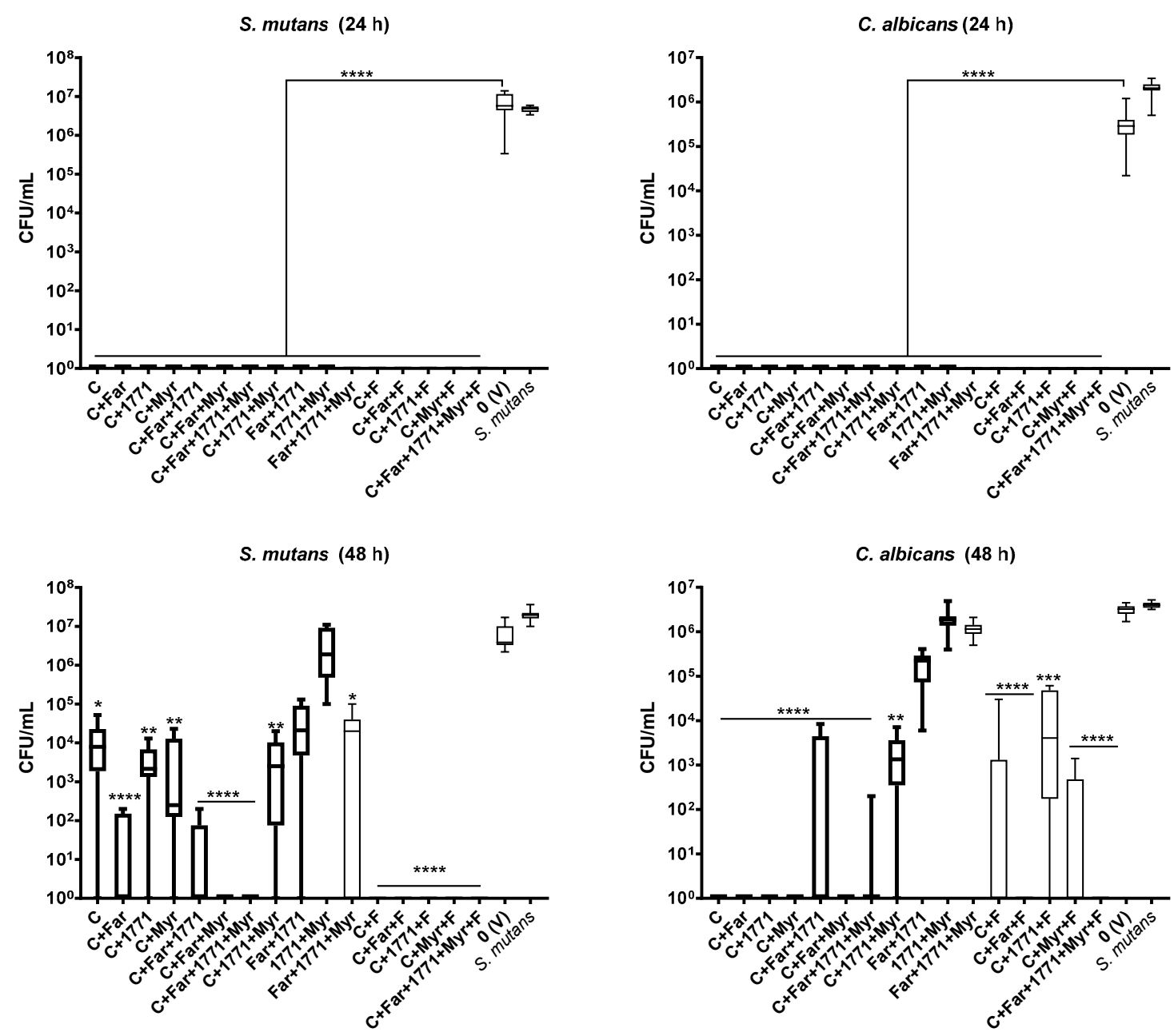

Figure 5. Antibiofilm activity of dual-species S. mutans and C. albicans biofilms with combined compounds (with and without sodium fluoride): C135 (C), tt-farnesol (Far), myricetin (Myr), 1771, and sodium fluoride (F). The top graphs presented data of $24 \mathrm{~h}$ biofilm (initial biofilm formation). The bottom graphs depict data of pre-formed biofilms (48 h). The data represents median and interquartile ranges $(n=9)$. Statistical differences are represented with * $(p=0.04),{ }^{* *}(p \leq 0.002)$, $* * *(p=0.0007)$, and ${ }^{* * *}(p \leq 0.0001)$ (Kruskal Wallis, followed by Dunn's test). The tabulated results are in Table S5.

\section{Discussion}

Several strategies can be used to control biofilms to prevent oral diseases. The classical strategies for oral biofilm control include brushing/flossing (mechanical removal of biofilms) and restricting dietary sugar intake (mainly frequency) to prevent biofilm build-up. Both diet restriction and brushing/flossing require behavioral compliance, which can be challenging. In addition, fluoride is used to avoid teeth demineralization and promote remineralization as part of oral hygiene products (toothpaste and mouthwashes) and/or supplied in tap drinking water. However, they may not be appropriate to all individuals, such as those without adequate dexterity (e.g., young children, elderly, people with disabilities, people in ICUs), which may require supervision for brushing/flossing 
and aids to weaken the overall biofilm structure to facilitate its mechanical removal, or even substances to enhance biofilm control.

Single targets for biofilm prevention and antimicrobial control can be difficult and limit the treatment options. Thus, combining agents with distinct targets can be an effective approach to access different sites in biofilms, which present complex biological traits and protected niches $[1,19]$. Therefore, this study evaluated the antimicrobial and antibiofilm activities of six compounds [ $t$-farnesol, myricetin, two 2'-hydroxychalcones (AGN and R815), 4'-hydroxychalcone (C135), and compound 1771] with different targets against $C$. albicans and $S$. mutans single- and dual-species settings.

Of note, an antimicrobial substance/molecule may not be an antibiofilm agent, and a compound with antibiofilm activity may not be an antimicrobial per se (e.g., molecules that affect extracellular enzymes responsible for the extracellular matrix construction). This scenario is depicted by the findings in both antimicrobial and antibiofilm assays performed as some agents were effective against the microorganisms in planktonic cultures and were not in the biofilms' settings. Also, the effect of compounds was mostly non-dose-dependent for both C. albicans and S. mutans.

The antimicrobial outcome for $S$. mutans showed the lowest $\mathrm{IC}_{50}$ value for compound $1771(7.813 \mu \mathrm{g} / \mathrm{mL})$, followed by $t$-farnesol $(31.25 \mu \mathrm{g} / \mathrm{mL}), \mathrm{C} 135(62.5 \mu \mathrm{g} / \mathrm{mL})$, while the highest value was observed for myricetin $(500 \mu \mathrm{g} / \mathrm{mL})$. However, $\mathrm{IC}_{50}$ values for $\mathrm{C}$. albicans were C135 $(62.5 \mu \mathrm{g} / \mathrm{mL})$ and $t t$-farnesol $(125 \mu \mathrm{g} / \mathrm{mL})$. Furthermore, $t t$-farnesol eliminated $\mathrm{CFU} / \mathrm{mL}$ count of both species reaching a MBC $(62.5 \mu \mathrm{g} / \mathrm{mL})$ and a MFC $(1000 \mu \mathrm{g} / \mathrm{mL})$. The compound 1771 presented MBC $(250 \mu \mathrm{g} / \mathrm{mL})$ and C135 reached MFC $(62.5 \mu \mathrm{g} / \mathrm{mL})$. The findings for $\mathrm{C} 135$ for both species and 1771 for $C$. albicans are presented for the first time here. Thus, C135 presented a promising antimicrobial effect for both species, and compound 1771 did not inhibit C. albicans growth.

A previous study with $S$. mutans using different concentrations of compound 1771 did not eliminate the bacterium [30], but the total elimination of CFU/mL count was observed here using the greatest concentration. In the same study [30], the antimicrobial activity of myricetin for $S$. mutans was at a lower concentration than the one found here. The antimicrobial effect of $t t$-farnesol on both species corroborates previous findings [18,22]. Among the three chalcones tested, the antimicrobial activity was better for C135, a $4^{\prime}$ hydroxychalcone. The other two 2'-hydroxychalcones (AGN and R815) did not present antimicrobial effect for $S$. mutans. These results can be explained by the differences between the chemical structure of the selected hydroxychalcones, suggesting that the presence of hydroxyl groups on the ring of the $4^{\prime}$-hydroxychalcone scaffold is crucial for the growth inhibition [24,38,39].

The presence of an extracellular matrix is essential for the existence of biofilm and the complete expression of virulence by microbial pathogens and pathobionts, hindering the action of antimicrobial agents and preventing their access to microbial cells [40]. Sucrose can modulate microbial synergism and ecology of the oral microbiota because its hexoses (glucose and fructose) are used for exopolysaccharides and organic acid production that influences the structure and composition of dental biofilms [1,6]. Cariogenic biofilms promote interactions and mechanisms that control dysbiosis [1,6] as observed on dualspecies biofilms of $S$. mutans and C. albicans in vitro [2,3]. Therefore, it is important to understand the mechanisms of possible antibiofilm compounds.

Myricetin and some hydroxychalcones inhibit S. mutans F-ATPase activity (acid tolerance mechanism) [19], glycolysis (organic acid production or acidogenicity) [19], and synthesis of extracellular matrix glucans (by interfering with $g t f s$ gene expression and Gtfs activity) $[19,24]$. The deficit in glucan production can compromise the integrity and 3D structure biofilms [4], facilitating their disruption. These findings can explain the antibiofilm effect of myricetin on S. mutans initial biofilms $(24 \mathrm{~h})$ and the greatest potential of C135 (4'-hydroxychalcone) on initial (24 h) and mature $S$. mutans biofilm (48 h). The weaker inhibition of myricetin on pre-formed biofilms ( $48 \mathrm{~h}$-biofilm eradication) can be promoted by the presence of pre-formed microcolonies and their 3D extracellular matrix. 
$t t$-farnesol eliminated the bacterium in 24- and 48 h-old single-species biofilms (at $62.5 \mu \mathrm{g} / \mathrm{mL}$ and higher concentrations). The antimicrobial and antibiofilm effect of this compound can be related to the targets on $S$. mutans cytoplasmatic membrane, altering its proton permeability, decreasing its tolerance to acid stress [18]. Compound 1771 also had a promising antibiofilm effect for the bacterium, especially on initial biofilm ( 24 h-old single-species biofilms). This compound inhibits the LTA synthesis [28], interfering with the cell wall composition, making the cytoplasmatic membrane an easy target for environmental stresses. Also, LTA from the cell wall are released in the matrix and interact with exopolysaccharides during the development and maturation of biofilms [41]; hence, interfering with LTA metabolism can impair cell wall and extracellular matrix composition. Thus, $t t$-farnesol and compound 1771 hindered $S$. mutans biofilms by promoting antimicrobial and antibiofilm activities.

Hydroxychalcones inhibit the cell wall formation of C. albicans cells [42,43], while ttfarnesol keeps the fungus in its yeast form [21]. However, it is unknown whether myricetin inhibits C. albicans biofilm formation or its extracellular matrix development or whether compound 1771 could target this fungus or its metabolism. The effect on the extracellular matrix components and construction could make the fungal cells more susceptible to antimicrobial agents. Here, single-species $C$. albicans biofilms were not greatly affected by myricetin. However, the $4^{\prime}$-hydroxychalcone $\mathrm{C} 135$ presented a promising effect in the initial $(24 \mathrm{~h})$ and mature $(48 \mathrm{~h})$ fungal biofilms. Also, $t t$-farnesol and 1771 were effective on initial fungal biofilm, but only $t t$-farnesol eliminated the fungus (at $62.5 \mu \mathrm{g} / \mathrm{mL}$ and higher concentrations). In addition, $t$-farnesol had a weak effect, while 1771 had practically no effect on fungal growth on pre-formed biofilms. Thus, eradication of C. albicans biofilm (48 h) was achieved only with C135.

Previous studies with $C$. albicans biofilms demonstrate that chalcones inhibited enzymes involved in resistance pathways [42]. Thus, the effect of C135 on C. albicans biofilms can be related to targets on resistance pathways; nevertheless, this hypothesis must be better explained. In addition, as observed on the data above from antimicrobial activity of $t$-farnesol, this compound can inhibit fungal growth, induce apoptosis in C. albicans cells, and inhibit the fungal hyphae [21,22]. As described for initial biofilms $(24 \mathrm{~h})$, the treatment was applied at $0 \mathrm{~h}$, so it may be that the effect of $t t$-farnesol (preventing filamentous morphology) supports the inhibition of fungal growth in $24 \mathrm{~h}$ while hampered its effect on pre-formed biofilm $(48 \mathrm{~h})$. Therefore, combine compounds could improve the effect in mature biofilms. This hypothesis is confirmed by the potentiated effect on $C$. albicans cells in dual-species biofilm $(48 \mathrm{~h}$ ) when $\mathrm{C} 135$ and $t t$-farnesol were combined, suggesting inhibition of the resistance mechanisms when both compounds are present.

Altogether, the data from antimicrobial and single-species biofilms assays enabled the selection of specific concentrations of the four compounds (C135, myricetin, $t t$-farnesol, and compound 1771) that were combined, with or without sodium fluoride, to assess the antibiofilm activity of formulations against dual-species $S$. mutans and C. albicans biofilm. All formulations without fluoride [(C135, C135+tt-farnesol, C135+1771, C135+myricetin, C135+tt-farnesol+1771, C135+tt-farnesol+myricetin, C135+tt-farnesol+1771+myricetin, C135+ $1771+$ myricetin, $t t$-farnesol+1771, 1771+myricetin, $t t$-farnesol+1771+myricetin) ] and with fluoride [(C135+fluoride, C135+tt-farnesol+fluoride, C135+1771+fluoride, C135+J10595+fluoride, C135+tt-farnesol+1771+myricetin+fluoride completely inhibited the initial biofilm formation $(24 \mathrm{~h})$. These findings showed that there might be a potential synergism between the compounds and a greater effect when they are combined and applied since the beginning of biofilm formation and during the $24 \mathrm{~h}$ of dual-species biofilm development. Part of the effect can be because of the antimicrobial effect per se, as microbial cells were exposed to formulations in their free form before adhesion to the surface. Also, the effect on extracellular matrix formation can not be ruled out.

In contrast, dual-species biofilm eradication $(48 \mathrm{~h})$ in which both species did not grow occurred for three formulations: C135+tt-farnesol+myricetin, C135+tt-farnesol+fluoride, and $C 135+t t$-farnesol+1771+myricetin+fluoride. Four formulations only eradicated the 
bacterial growth (C135+tt-farnesol+1771+myricetin, C135+fluoride, C135+1771+fluoride, and C135+myricetin+fluoride), while other four formulations eradicated fungal growth (C135, C135+tt-farnesol, C135+1771, and C135+myricetin). Of note, the formulations with fluoride exhibited a greater antibiofilm activity (mainly for the bacterium), reinforcing the importance of its inclusion in strategies to prevent and control cariogenic biofilms [18]; fewer and less severe carious lesions were observed using combined treatments and fluoride on a rodent model of dental caries [19]. Fluoride can interfere with microbial metabolism, especially on glycolytic enzymes and proton gradient dissipation on the cell cytoplasmatic membrane (when the extracellular $\mathrm{pH}$ is higher than the intracellular $\mathrm{pH}$ ) [44]. This effect can hamper cell growth. Nevertheless, C135 alone or combined with other agents (even those without pronounced effect on singles-species $48 \mathrm{~h}$-old biofilms) prevented fungal and bacterial growth in dual-species biofilms, making it a promising agent.

The present findings provided insights about: (i) compounds as inhibitors of biofilm formation of single-species biofilm ( $24 \mathrm{~h}$ ); (ii) compounds that can eradicate pre-formed biofilm ( $48 \mathrm{~h}$ ); and (iii) formulations with combined compounds for biofilm inhibition ( $24 \mathrm{~h}$ ) and eradication ( $48 \mathrm{~h}$ ) of both species in dual-species biofilms. C135 is a novel compound with possible distinct targets alone or in combination with other agents. The formulations that combined agents with distinct targets prevented C. albicans and S. mutans dual-species biofilm build-up in vitro. The formulation $\mathrm{C} 135+t t$-farnesol with or without fluoride may represent a potential alternative approach that deserves further investigation, including cytotoxicity to host $[30,45]$. Therefore, the outcomes of this study could be applied to future studies using the compound alone or combined as an adjuvant strategy to control oral biofilms using shorter exposure times, as mouthwashes.

Supplementary Materials: The following are available online at https:/ / www.mdpi.com/article/10 .3390/jof7050340/s1. Table S1: Antimicrobial activity of S. mutans using six compounds: C135, R815, AGN, $t t$-farnesol, myricetin, and 1771. Data shown in Figure 1 as CFU/mL. Table S2: Antimicrobial activity of $C$. albicans with compounds: C135, $t$-farnesol, myricetin, and 1771 . Data shown in Figure 2 as CFU/mL. Table S3: Antibiofilm activity of $S$. mutans with compounds: C135, $t t$-farnesol, myricetin, and 1771 of $24 \mathrm{~h}$ biofilm (initial biofilm formation) and pre-formed biofilms ( $48 \mathrm{~h}$ ). Data shown in Figure 3 as CFU/mL. Table S4: Antibiofilm activity of C. albicans with compounds: C135, $t t$-farnesol, myricetin, and 1771 of $24 \mathrm{~h}$ biofilm (initial biofilm formation) and pre-formed biofilms ( $48 \mathrm{~h}$ ). Data shown in Figure 4 as CFU/mL. Table S5. Antibiofilm activity of dual-species S. mutans and C. albicans biofilms with combined compounds (with and without sodium fluoride): C135 (C), tt-farnesol (Far), myricetin (Myr), 1771, and sodium fluoride (F). Data shown in Figure 5 as CFU/mL.

Author Contributions: Conceived and designed the experiments: C.I.V.L. and M.I.K. Carried out the experiments: C.I.V.L. Analyzed the data C.I.V.L. and M.I.K. Reagents/materials/analysis tools: M.I.K. Wrote the paper: C.I.V.L., A.C.U.d.A.L., and M.I.K. All authors have read and agreed to the published version of the manuscript.

Funding: This research was supported by a research grant from the National Council for Scientific and Technological Development (CNPq \#409668/2018-4 to M.I.K.) and scholarships (the Coordination of Superior Level Staff Improvement-CAPES \#001 and CNPq \#141316/2020-9 to C.I.V.L.; and CAPES \#001 and the São Paulo Research Foundation-FAPESP \#2020/02946-2 to A.C.U.A.L.). The funding body had no role in the design of the study or collection, analysis, and interpretation of data and in writing the manuscript.

Institutional Review Board Statement: Not applicable.

Informed Consent Statement: Not applicable.

Data Availability Statement: The data presented in this study are available as supplementary material. Additional data are available on request from the corresponding author.

Acknowledgments: The authors are thankful to CNPq, CAPES, and FAPESP for grant and scholarships funding. The present research will be part of the Ph.D. thesis by C.I.V.L.

Conflicts of Interest: The authors declare that the research was conducted in the absence of any commercial or financial relationships that could be construed as a potential conflict of interest. 


\section{References}

1. Lamont, R.J.; Koo, H.; Hajishengallis, G. The oral microbiota: Dynamic communities and host interactions. Nat. Rev. Microbiol. 2018, 16, 745-759. [CrossRef]

2. Lobo, C.I.V.; Rinaldi, T.B.; Christiano, C.M.S.; Leite, L.S.; Barbugli, P.A.; Klein, M.I. Dual-species biofilms of Streptococcus mutans and Candida albicans exhibit more biomass and are mutually beneficial compared with single-species biofilms. J. Oral Microbiol. 2019, 11, 1581520. [CrossRef]

3. Falsetta, M.L.; Klein, M.I.; Colonne, P.M.; Scott-Anne, K.; Gregoire, S.; Pai, C.H.; Gonzalez-Begne, M.; Watson, G.; Krysan, D.J.; Bowen, W.H.; et al. Symbiotic relationship between Streptococcus mutans and Candida albicans synergizes virulence of plaque biofilms in vivo. Infect. Immun. 2014, 82, 1968-1981. [CrossRef] [PubMed]

4. Bowen, W.H.; Koo, H. Biology of Streptococcus mutans Derived Glucosyltransferases: Role in Extracellular Matrix formation of Cariogenic Biofilms. Caries Res. 2011, 45, 69-86. [CrossRef]

5. Takahashi, N.; Nyvad, B. The role of bacteria in the caries process: Ecological perspectives. J. Dent. Res. 2011, 90, 294-303. [CrossRef]

6. Bowen, W.H.; Burne, R.A.; Wu, H.; Koo, H. Oral Biofilms: Pathogens, Matrix, and Polymicrobial Interactions in Microenvironments. Trends Microbiol. 2018, 26, 229-242. [CrossRef] [PubMed]

7. Gregoire, S.; Xiao, J.; Silva, B.B.; Gonzalez, I.; Agidi, P.S.; Klein, M.I.; Ambatipudi, K.S.; Rosalen, P.L.; Bauserman, R.; Waugh, R.E.; et al. Role of glucosyltransferase B in the interactions of Candida albicans with Streptococcus mutans and experimental pellicle formed on hydroxyapatite surface. Appl. Environ. Microbiol. 2011, 77, 6357-6367. [CrossRef] [PubMed]

8. Klinke, T.; Kneist, S.; De Soet, J.; Kuhlisch, E.; Mauersberger, S.; Förster, A.; Klimm, W. Acid production by oral strains of Candida albicans and lactobacilli. Caries Res. 2009, 43, 83-91. [CrossRef]

9. Al-Fattani, M.A.; Douglas, L.J. Biofilm matrix of Candida albicans and Candida tropicalis: Chemical composition and role in drug resistance. J. Med. Microbiol. 2006, 55, 999-1008. [CrossRef]

10. Lal, P.; Sharma, D.; Pruthi, P.; Pruthi, V. Exopolysaccharide analysis of biofilm-forming Candida albicans. J. Appl. Microbiol. 2010, 109, 128-136. [CrossRef]

11. Nett, J.E.; Sanchez, H.; Cain, M.T.; Andes, D.R. Genetic basis of Candida biofilm resistance due to drug-sequestering matrix glucan. J. Infect. Dis. 2010, 202, 171-175. [CrossRef]

12. Zarnowski, R.; Westler, W.M.; Lacmbouh, G.A.; Marita, J.M.; Bothe, J.R.; Bernhardt, J.; Sahraoui, A.L.-H.; Fontaine, J.; Sanchez, H.; Hatfield, R.D.; et al. Novel entries in a fungal biofilm matrix encyclopedia. mBio 2014, 5, e01333-14. [CrossRef]

13. Taff, H.T.; Nett, J.E.; Zarnowski, R.; Ross, K.M.; Sanchez, H.; Cain, M.T.; Hamaker, J.; Mitchell, A.P.; Andes, D.R. A Candida Biofilm-Induced Pathway for Matrix Glucan Delivery: Implications for Drug Resistance. PLoS Pathog. 2012, 8, e1002848. [CrossRef]

14. Mitchell, K.F.; Zarnowski, R.; Sanchez, H.; Edward, J.A.; Reinicke, E.L.; Nett, J.E.; Mitchell, A.P.; Andes, D.R. Community participation in biofilm matrix assembly and function. Proc. Natl. Acad. Sci. USA 2015, 112, 4092-4097. [CrossRef] [PubMed]

15. Melvaer, K.L.; Helgeland, K.; Rolla, G. Some physical and chemical properties of 'soluble' and 'insoluble' polysacharides produced by strains of Streptococcus mutans and sanguis. Caries Res. 1972, 6, 79. [PubMed]

16. Brookes, Z.L.S.; Bescos, R.; Belfield, L.A.; Ali, K.; Roberts, A. Current uses of chlorhexidine for management of oral disease: A narrative review. J. Dent. 2020, 103, 103497. [CrossRef]

17. Ten Cate, J.M. Novel anticaries and remineralizing agents: Prospects for the future. J. Dent. Res. 2012, 91, 813-815. [CrossRef] [PubMed]

18. Koo, H.; Schobel, B.; Scott-Anne, K.; Watson, G.; Bowen, W.H.; Cury, J.A.; Rosalen, P.L.; Park, Y.K. Apigenin and tt-farnesol with fluoride effects on S. mutans biofilms and dental caries. J. Dent. Res. 2005, 84, 1016-1020. [CrossRef]

19. Falsetta, M.L.; Klein, M.I.; Lemos, J.A.; Silva, B.B.; Agidi, S.; Scott-Anne, K.K.; Koo, H. Novel antibiofilm chemotherapy targets exopolysaccharide synthesis and stress tolerance in Streptococcus mutans to modulate virulence expression in vivo. Antimicrob. Agents Chemother. 2012, 56, 6201-6211. [CrossRef]

20. Bersan, S.M.F.; Galvão, L.C.C.; Goes, V.F.F.; Sartoratto, A.; Figueira, G.M.; Rehder, V.L.G.; Alencar, S.M.; Duarte, R.M.T.; Rosalen, P.L.; Duarte, M.C.T. Action of essential oils from Brazilian native and exotic medicinal species on oral biofilms. BMC Complement. Altern. Med. 2014, 14, 451. [CrossRef]

21. Hall, R.A.; Turner, K.J.; Chaloupka, J.; Cottier, F.; De Sordi, L.; Sanglard, D.; Levin, L.R.; Buck, J.; Mühlschlegel, F.A. The quorum-sensing molecules farnesol/homoserine lactone and dodecanol operate via distinct modes of action in Candida albicans. Eukaryot. Cell 2011, 10, 1034-1042. [CrossRef]

22. Zhu, J.; Krom, B.P.; Sanglard, D.; Intapa, C.; Dawson, C.C.; Peters, B.M.; Shirtliff, M.E.; Jabra-Rizk, M.A. Farnesol-Induced Apoptosis in Candida albicans Is Mediated by Cdr1-p Extrusion and Depletion of Intracellular Glutathione. PLoS ONE 2011, 6, e28830. [CrossRef]

23. Rocha, G.R.; Florez Salamanca, E.J.; de Barros, A.L.; Lobo, C.I.V.; Klein, M.I. Effect of $t$-farnesol and myricetin on in vitro biofilm formed by Streptococcus mutans and Candida albicans. BMC Complement. Altern. Med. 2018, 18, 61. [CrossRef]

24. Nijampatnam, B.; Casals, L.; Zheng, R.; Wu Hui Velu, S.E. Hydroxychalcone inhibitors of Streptococcus mutans glucosyl transferases and biofilms as potential anticaries agents. Bioorg. Med. Chem. Lett. 2016, 26, 3508-3513. [CrossRef] [PubMed]

25. Sato, M.; Tsuchiya, H.; Akagiri, M.; Takagi, N.; Iinuma, M. Growth inhibition of oral bacteria related to denture stomatitis by anti-candidal chalcones. Aust. Dent. J. 1997, 42, 343-346. [CrossRef] [PubMed] 
26. Aboody, M.S.A.; Mickymaray, S. Anti-Fungal Efficacy and Mechanisms of Flavonoids. Antibiotics 2020, 9, 45. [CrossRef] [PubMed]

27. Tsuchiya, H.; Sato, M.; Akagiri, M.; Takagi, N.; Tanaka, T.; Iinuma, M. Anti-Candida activity of synthetic hydroxychalcones. Die Pharm. 1994, 49, 756-758.

28. Richter, S.G.; Elli, D.; Kim, H.K.; Hendrickx, A.P.A.; Sorg, J.A.; Schneewind, O.; Missiakas, D. Small molecule inhibitor of lipoteichoic acid synthesis is an antibiotic for Gram-positive bacteria. Proc. Natl. Acad. Sci. USA 2013, 110, 3531-3536. [CrossRef] [PubMed]

29. Paganelli, F.L.; Van De Kamer, T.; Brouwer, E.C.; Leavis, H.L.; Woodford, N.; Bonten, M.J.; Willems, R.J.; Hendrickx, A.P. Lipoteichoic acid synthesis inhibition in combination with antibiotics abrogates growth of multidrug-resistant Enterococcus faecium. Int. J. Antimicrob. Agents 2017, 49, 355-363. [CrossRef] [PubMed]

30. Castillo Pedraza, M.C.; Fratucelli, E.D.O.; Ribeiro, S.M.; Florez Salamanca, E.J.; Colin, J.S.; Klein, M.I. Modulation of lipoteichoic acids and exopolysaccharides prevents Streptococcus mutans biofilm accumulation. Molecules 2020, 25, 2232. [CrossRef]

31. Clinical and Laboratory Standards Institute. CLSI Document M100-S25. In Performance Standards for Antimicrobial Susceptibility Testing; Clinical and Laboratory Standards Institute: Wayne, PA, USA, 2015.

32. Clinical and Laboratory Standards Institute. CLSI Document M27: Reference Method for Broth Dilution Antifungal Susceptibility Testing of Yeasts. In Performance Standards for Antimicrobial Susceptibility Testing, 4th ed.; Clinical and Laboratory Standards Institute: Wayne, PA, USA, 2017.

33. Clinical and Laboratory Standards Institute. CLSI Document M23: Development of In Vitro Susceptibility Testing Criteria and Quality Control Parameters. In Performance Standards for Antimicrobial Susceptibility Testing, 5th ed.; Clinical and Laboratory Standards Institute: Wayne, PA, USA, 2018.

34. Van Dijck, P.; Sjollema, J.; Cammue, B.P.A.; Lagrou, K.; Berman, J.; D’Enfert, C.; Andes, D.R.; Arendrup, M.C.; Brakhage, A.A.; Calderone, R.; et al. Methodologies for in vitro and in vivo evaluation of efficacy of antifungal and antibiofilm agents and surface coatings against fungal biofilms. Microb. Cell 2018, 5, 300-326. [CrossRef] [PubMed]

35. Eloff, J.N. Avoiding pitfalls in determining antimicrobial activity of plant extracts and publishing the results. BMC Complement. Altern. Med. 2019, 19, 106. [CrossRef]

36. Saputo, S.; Faustoferri, R.C.; Quivey, R.G., Jr. A drug repositioning approach reveals that Streptococcus mutans is susceptible to a diverse range of established antimicrobials and nonantibiotics. Antimicrob. Agents Chemother. 2017, 62, e01674-17. [CrossRef] [PubMed]

37. Zero, D.T. Dentifrices, mouthwashes, and remineralization/caries arrestment strategies. BMC Oral Health 2006, 6 (Suppl. S1), S9. [CrossRef]

38. Heim, K.E.; Tagliaferro, A.R.; Bobilya, D.J. Flavonoid antioxidants: Chemistry, metabolism and structure-activity relationships. J. Nutr. Biochem. 2002, 13, 572-584. [CrossRef]

39. Cushnie, T.P.; Lamb, A.J. Antimicrobial activity of flavonoids. Int. J. Antimicrob. Agents 2005, 26, 343-356. [CrossRef]

40. Flemming, H.C.; Wingender, J. The biofilm matrix. Nat. Rev. Microbiol. 2010, 8, 623-633. [CrossRef]

41. Castillo Pedraza, M.C.; Rosalen, P.L.; de Castilho, A.R.F.; Freires, I.D.A.; Leite, L.D.S.; Leite, L.D.S.; Faustoferri, R.C.; Quivey, R.G., Jr.; Klein, M.I. Inactivation of Streptococcus mutans genes lytST and dltAD impairs its pathogenicity in vivo. J. Oral Microbiol. 2019, 11, 1607505. [CrossRef]

42. Batovska, D.; Parushev, S.; Slavova, A.; Bankova, V.; Tsvetkova, I.; Ninova, M.; Najdenski, H. Study on the substituents' effects of a series of synthetic chalcones against the yeast Candida albicans. Eur. J. Med. Chem. 2007, 42, 87-92. [CrossRef] [PubMed]

43. Santana, D.P.; Ribeiro, T.F.; Ribeiro, E.L.; de Aquino, G.L.B.; Faleiro Naves, P.L. Ação de chalconas contra a formação de biofilme de Candida albicans. J. Basic Appl. Pharm. Sci. 2015, 36, 83-90.

44. Marquis, R.E.; Clock, S.A.; Mota-Meira, M. Fluoride and organic weak acids as modulators of microbial physiology. FEMS Microbiol. Rev. 2003, 26, 493-510. [CrossRef] [PubMed]

45. Seleem, D.; Benso, B.; Noguti, J.; Pardi, V.; Murata, R.M. In Vitro and In Vivo Antifungal Activity of Lichochalcone-A against Candida albicans Biofilms. PLoS ONE 2016, 11, e0157188. [CrossRef] [PubMed] 